\title{
Optically driven micropump produced by three-dimensional two-photon microfabrication
}

\author{
Shoji Maruo a) and Hiroyuki Inoue \\ Department of Mechanical Engineering, Graduate School of Engineering, Yokohama National University, \\ 79-5 Tokiwadai, Hodogaya-ku, Yokohama 240-8501, Japan
}

(Received 23 May 2006; accepted 15 August 2006; published online 2 October 2006)

\begin{abstract}
An optically driven lobed micropump was developed using three-dimensional two-photon microfabrication. The two built-in rotors, $9 \mu \mathrm{m}$ in diameter, are cooperatively driven by means of time-divided scanning of a single laser beam. It was demonstrated that a tracer particle was moved by simultaneously rotating the two rotors. The velocity of the tracer particle was proportional to the rotation speed of the rotors in the range of $0.2-0.7 \mu \mathrm{m} / \mathrm{s}$. The flow rate was estimated to be sub-pL/min level. This ultralow flow rate will be useful for further integration and miniaturization of micro-total-analysis systems. (C) 2006 American Institute of Physics.
\end{abstract}

[DOI: $10.1063 / 1.2358820$ ]

Various types of microfluidic chips are being developed for DNA analysis and proteomics research. ${ }^{1,2}$ Most currently used microfluidic chips utilize electrophoresis to transport fluids and samples. This technique merely requires the insertion of electrodes inside a microchannel to ensure precisely controlled transport of liquids and samples. For that reason, microfluidic chips based on electrophoresis are simple, cheap, and disposable. However, it has not been proven possible to extend electrophoretic techniques to chemical synthesis and analytic processes, since usable liquids and samples are limited to electrolytes. Built-in micropumps based on pressure-driven flow are thus necessary.

In the past two decades, various kinds of pressure-driven micropumps have been developed using a range of micromachining techniques. ${ }^{3,4}$ Most of the previously developed micropumps are diaphragm types, in which piezoelectric actuators or pneumatic actuation are utilized to deform an elastic membrane that drives fluid transport. To obtain adequate displacement of the membrane, the diameter of the membrane must range from 100 to $500 \mu \mathrm{m}$. As a result, the overall dimensions of the micropumps are much larger than the microchannels. This prevents the further miniaturization and integration of microfluidic components. In addition, the usage of built-in high-precision microactuators makes biochips expensive. For these reasons, most current disposable microfluidic chips for chemical synthesis and analysis still utilize external pumps such as syringe types rather than built-in micropumps. 5

As a promising method of addressing the above issues, several kinds of integrated optically driven microfluidic systems utilizing microparticles suspended in liquid have recently been reported. ${ }^{6-8}$ These optically driven microfluidic devices make it possible to pump not only electrolytes but also organic and inorganic solvents. In addition, the optical driving of microfluidic elements provides disposable functional biochips without the need for expensive actuators and sensors. However, the previously reported optically driven microfluidic systems are difficult to apply to practical and automatic analytic systems. This is because numerous microparticles must be introduced and positioned at fixed locations on the chip. These time-consuming and complicated pro-

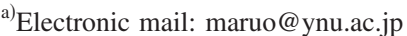

cesses impede the practical application of optically controlled microfluidic devices. In addition, suspended microparticles cannot be confined at their individual appointed places without continuous irradiation of light. This limits the parallel and multifunctional operations of chemical analysis and synthesis by use of laser scanning micromanipulation techniques.

Here we report an entirely optically driven micropump, in which two lobed rotors are incorporated into a microchannel. Unlike microparticle-based microfluidic devices, the lobed rotors are individually confined to a microchannel by their own shaft, thus preventing the rotors from moving unless continuously irradiated with light. This allows the micropump to be easily integrated into a practical biochip. Figure 1(a) shows a schematic diagram of the optically driven lobed micropump. This micropump is driven by means of radiation pressure generated by focusing a laser beam. When the laser beam is focused on the side of the rotor, the net radiation pressure applied to the rotor points toward the fo-

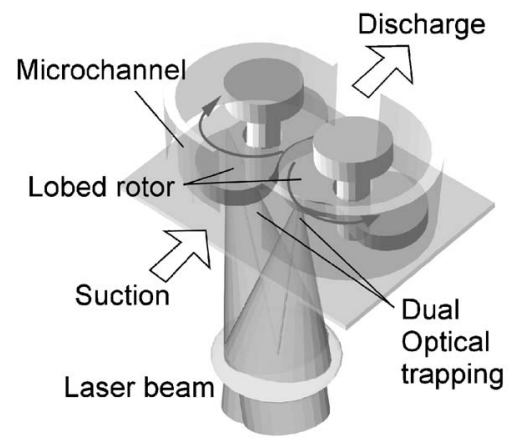

(a)

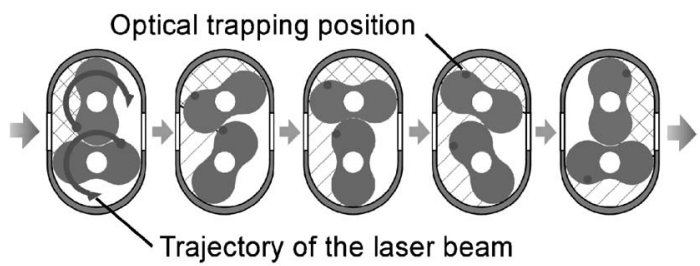

(b)

FIG. 1. Optically driven lobed micropump. (a) Schematic diagram of the lobed micropump driven by the time-divided laser scanning of a single laser beam. (b) Fluid transport by optically rotating two rotors. 


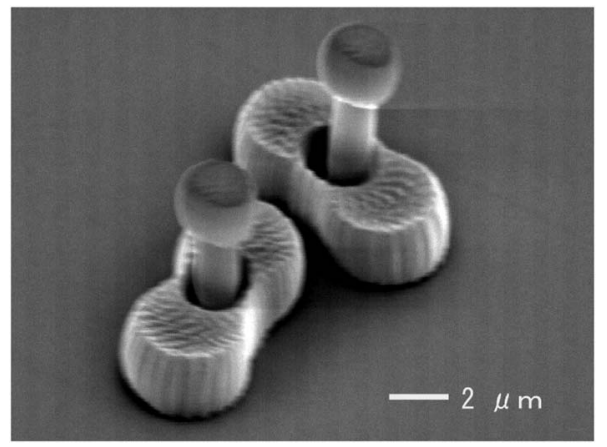

FIG. 2. Scanning electron microscopic image of a prototype of the lobed micropump.

cus, allowing the rotor to be controlled by changing the trajectory of the scanning laser beam.

To simultaneously control two rotors with a single laser beam, there are several feasible techniques such as holographic optical tweezers, ${ }^{9,10}$ time-divided laser scanning methods, ${ }^{11,12}$ and a patterned optical field formation technique. ${ }^{13}$ In particular, the time-divided laser scanning method offers sufficient flexibility and throughput by using a simple and low-cost system with high-speed scanners such as galvano mirrors and piezoelectric actuators. As shown in Fig. 1(b), therefore, we utilized the time-divided laser scanning method to control the two rotors simultaneously. The black points shown in Fig. 1(b) are trapping points for each status. The laser beam is divided and scanned along two circular trajectories in the opposite direction. In this case, on-off control of the laser beam is not performed when the laser beam alternates between the two trajectories. However, the intensity distribution along the target trajectories is high enough to rotate the rotors smoothly by optimizing the repetition frequency of the laser beam moving between the two trajectories. While the rotors are tightly engaged and rotated, fluid is trapped in the spaces between the rotors in the periphery of both rotors and carried around in them, as shown in Fig. 1(b). Since the meshed rotors serve as a seal between inlet and outlet, the two rotors need to be meshed tightly by radiation pressure while the laser beam is scanning. In addition, close tolerances must be maintained between the housing and the rotor sides and periphery to prevent excess leakage.

As a method of fabricating both rotors and a microchannel precisely, we employed a three-dimensional (3D) twophoton microfabrication technique. ${ }^{14,15}$ In the two-photon process, freely movable micromechanisms can be directly fabricated by scanning a femtosecond pulsed laser beam inside a photopolymer. Since the movable microparts are steadied by the viscous force of the photopolymer, no supporting columns or layers are required. ${ }^{16}$ By using the direct writing process for movable microparts, we have already succeeded in fabricating simple movable micromachines

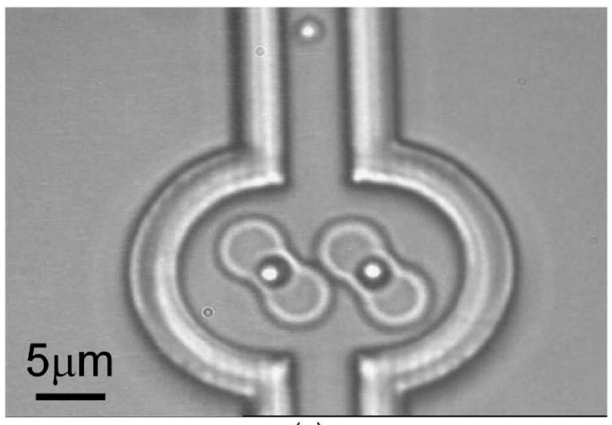

(a)

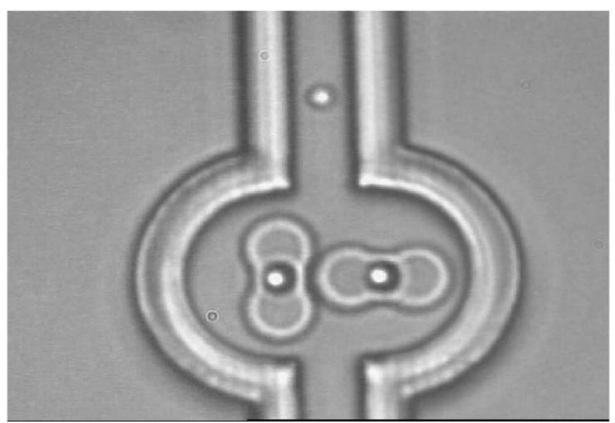

(b)

FIG. 4. Sequential images taken while driving the optically driven micropump.

such as single microgears and nanotweezers. ${ }^{17,18}$ However, tightly engaged micromachines, such as those required for high precision micropumps, have not been fabricated up to now.

To construct interlocking micromachines using the direct writing process, we first devised how to draw the movable parts. When a photopolymer is solidified by irradiation of a laser beam, presolidified parts are attracted to the solidifying parts as a result of the shrinkage that occurs during the photopolymerization process. This phenomenon makes individual movable parts put together during the laser writing process. Actually, if we draw the two rotors under the condition that they are engaged, the rotors cling together. On the other hand, if we fabricate two lobed rotors in parallel with the optimal clearance, the rotors do not stick together. After rinsing off the remaining unsolidified photopolymer, the rotors can be easily engaged and driven by means of the timedivided laser scanning manipulation technique.

Figure 2 shows a scanning electron microscopic image of a prototype of the lobed micropump. The length of the major axis of each rotor is $9 \mu \mathrm{m}$. The thickness of the rotor is $2.5 \mu \mathrm{m}$. The lobed rotors are incorporated into a microchannel whose inlet and outlet are in $5 \mu \mathrm{m}$ wide and $7 \mu \mathrm{m}$ high. Both the rotors and the microchannel are fabricated using a 3D two-photon microfabrication system developed by us. In our fabrication system, a titanium sapphire laser (wavelength: $752 \mathrm{~nm}$, pulse width: $200 \mathrm{fs,} \mathrm{repetition} \mathrm{rate:}$

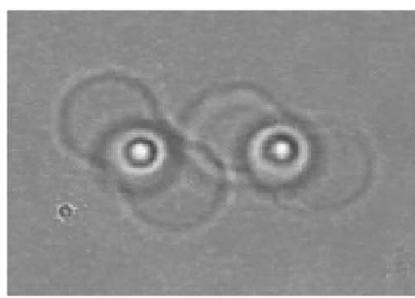

(a)

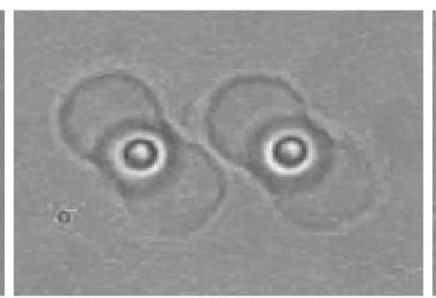

(b)

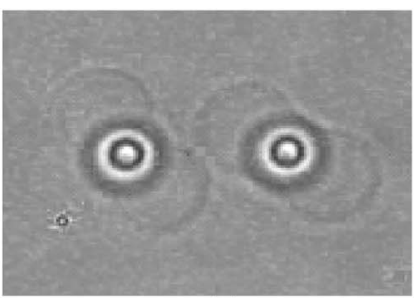

(c)
FIG. 3. Optical microscopic images of rotors with different center distances. (a) Center distance: $7 \mu \mathrm{m}$, (b) center distance: $7.5 \mu \mathrm{m}$, and (c) center distance: $8 \mu \mathrm{m}$.

\section{(-}




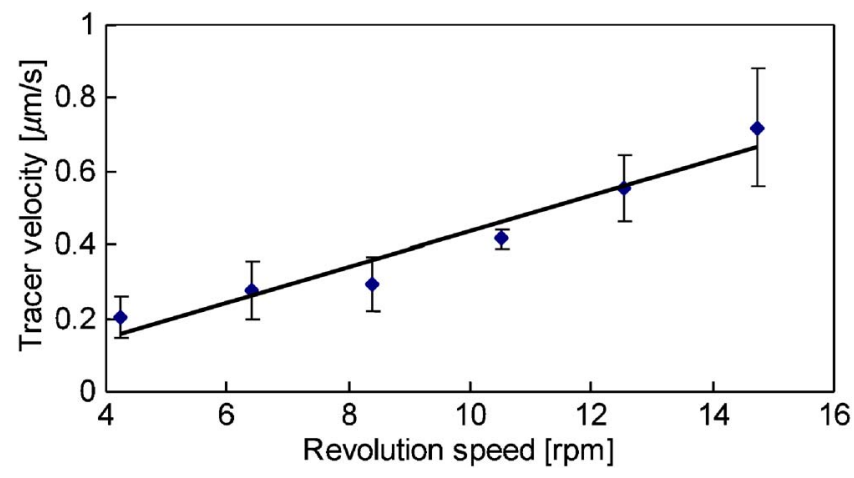

FIG. 5. (Color online) Dependence of the velocity of the tracer particle on the rotational speed of the rotors.

$76 \mathrm{MHz}$ ) was used to generate two-photon-absorbed photopolymerization. The femtosecond pulsed laser beam is collimated through the beam expander and focused inside a commercial available epoxy-type photopolymer (D-MEC Co., Ltd., SCR-701) with an oil immersion objective lens whose numerical aperture is 1.25. The laser beam is scanned in a focal plan by using galvano scanners (GSI Lumonics, Inc., M2 scanners) while the stage (Sigma Koki Co., Ltd, Mark 204) supporting the sample is lowered. After drawing the micromachine, unsolidified photopolymer is washed out with glycol ether ester. To make the micropump shown in Fig. 2, the laser power and scanning speed of the laser beam were $150 \mathrm{~mW}$ and $30 \mu \mathrm{m} / \mathrm{s}$, respectively. The laser power is measured in front of the beam expander. The pitch of each layer accumulated to make the microchannel and the rotors along the optical axis is $0.5 \mu \mathrm{m}$. The center distance between the rotors was experimentally determined to optimize the clearance between the rotors. Although the optimal clearance is primarily designed using a 3D computer aided design system, the fabricated rotors are actually different in size owing to shrinkage during the photopolymerization process. For this reason, the center distance was optimized experimentally. In our experiments on optical driving, the optical system used for the 3D two-photon microfabrication was also applied with a Ti:sapphire laser operated in cw mode. Figures 3(a)-3(c) show optical microscopic images of the rotors with different center distances. As a result of verification experiments on optical driving with each pair of rotors, the center distance of $7.5 \mu \mathrm{m}$ allowed us to closely interlock the rotors.

Finally, we demonstrated that fluid was transported with the optically driven micropump including the pair of rotors with optimal center distance. Figures 4(a) and 4(b) show sequential images of the micropump being driven. A tracer particle was also fabricated in the microchannel using 3D two-photon microfabrication. The tracer particle was optically trapped to prevent it from moving around while unsolidified photopolymer was being washed out. After the washing process, the tracer particle was released, after which the rotors were interlocked using the time-divided laser scanning technique. As a result, the tracer particle was brought into the microchamber by the pumped flow. When the rotation direction was reversed, the tracer particle was pushed to the outlet. Figure 5 shows the dependence of the velocity of the tracer particle on the rotational speed of the rotors. It is clear that the velocity of the tracer particle is proportional to the rotational speed of the rotors. The flow rate was estimated at less than $1 \mathrm{pL} / \mathrm{min}$. Ultralow flow rates such as this give our Downloaded 01 Feb 2007 to 133.34.171.229. Redistribution subject mechanism potential for use in micro-nanofluidic devices. The clearance between the rotors and the chamber is one of the most important parameters in determining the pumping efficiency. We experimentally confirmed that the flow velocity is almost constant with a clearance of less than $3 \mu \mathrm{m}$. The micropump is suitable for the transportation of highly viscous fluid, since it is a kind of positive displacement pump. The viscous drag force exerted on the rotor is proportional to the rotation speed of the rotor in Stokes approximation for low Reynolds number flow. Therefore, when the rotation speed of the rotors is low, highly viscous fluids can be moved. The refractive indices of usable liquids are limited by that of the rotors, since the refractive index of the rotors must be higher than that of the surrounding liquid to trap the rotors. The use of photopolymers with high refractive indices will expand the range of usable liquids.

In summary, we have developed a lobed micropump driven by light using an assembly-free process by direct laser writing with a femtosecond laser beam. Since the micropump is constructed only of photopolymer and driven by light, it is ideal for disposable usage. In addition, the pulsation pressure of the lobed micropump is intrinsically smaller than that of conventional pressure-driven micropumps with a deformable membrane and check valves, making our lobed micropump suitable for continuous-flow-type microfluidic devices that are often used for chemical analysis and synthesis. ${ }^{5}$ Furthermore, by using 3D two-photon microfabrication, we can fabricate not only micropumps but also microtweezers inside a biochip. ${ }^{18}$ Optically controlled biochips of this type will be powerful tools for bionanotechnologies and medical applications.

This research was supported in part by research grants from the Japan Society for the Promotion of Science [Grantin-Aid for Young Scientists (A), Exploratory Research, and Scientific Research in Priority Areas: Systems Cell Engineering by Multi-Scale Manipulation].

${ }^{1}$ M. A. Burns, B. N. Johnson, S. N. Brahmasandra, K. Handique, J. R. Webster, M. Krishnan, T. S. Sammarco, P. M. Man, D. Jones, D. Heldsinger, C. H. Mastrangelo, and D. T. Burke, Science 282, 484 (1998). ${ }^{2}$ N. Lion, T. C. Rohner, L. Dayon, I. L. Arnaud, E. Damoc, N. Youhnovski, Z. Y. Wu, C. Roussel, J. Josserand, H. Jensen, J. S. Rossier, M. Przybylski, and H. H. Girault, Electrophoresis 24, 3533 (2003).

${ }^{3}$ S. Shoji and M. Esashi, J. Micromech. Microeng. 4, 157 (1994).

${ }^{4}$ D. J. Laser and J. G. Santiago, J. Micromech. Microeng. 14, R35 (2004).

${ }^{5}$ K. Sato, A. Hibara, M. Tokeshi, H. Hisamoto, and T. Kitamori, Adv. Drug Delivery Rev. 55, 379 (2003).

${ }^{6}$ A. Terray, J. Oakey, and D. W. M. Marr, Science 296, 1841 (2002).

${ }^{7}$ A. Terray, J. Oakey, and D. W. M. Marr, Appl. Phys. Lett. 81, 1555 (2002).

${ }^{8}$ J. Glückstad, Nat. Mater. 3, 9 (2004).

${ }^{9}$ J. Leach, G. Sinclair, P. Jordan, J. Courtial, M. J. Padgett, J. Cooper, and Z. J. Laczik, Opt. Express 12, 220 (2004).

${ }^{10}$ P. J. Rodrigo, V. R. Daria, and J. Glückstad, Appl. Phys. Lett. 86, 74103 (2005).

${ }^{11}$ F. Arai, K. Yoshikawa, T. Sakami, and T. Fukuda, Appl. Phys. Lett. 85, 4301 (2004).

${ }^{12}$ C. Mio, T. Gong, A. Terray, and D. W. M. Marr, Rev. Sci. Instrum. 71, 2196 (2000).

${ }^{13}$ K. Sasaki, M. Koshioka, H. Misawa, N. Kitamura, and H. Masuhara, Opt. Lett. 16, 1463 (1991).

${ }^{14}$ S. Maruo, O. Nakamura, and S. Kawata, Opt. Lett. 22, 132 (1997).

${ }^{15}$ S. Kawata, H.-B. Sun, T. Tanaka, and K. Takada, Nature (London) 412, 697 (2001)

${ }^{16}$ S. Maruo and K. Ikuta, Appl. Phys. Lett. 76, 2656 (2000).

${ }^{17}$ S. Maruo, K. Ikuta, and H. Korogi, J. Microelectromech. Syst. 12, 533 (2003).

${ }^{18}$ S. Maruo, K. Ikuta, and H. Korogi, Appl. Phys. Lett. 82, 133 (2003). 